NB: This is an author's final version of the paper published in Emotion Review April 2012 vol. 4 no. 2 197-202 doi: 10.1177/1754073911430139. This version may differ slightly from the published version and the pagination is different. The published version of the paper is available at http://emr.sagepub.com/content/4/2/197

\title{
Psychiatric classification and subjective experience
}

\section{Rachel Cooper}

\begin{abstract}
This paper does not directly consider the feelings and emotions that occur in mental illness. Rather it concerns a higher-level methodological question: to what extent is an analysis of feelings and felt emotions of importance for psychiatric classification? Some claim that producing a phenomenologically-informed descriptive psychopathology is a prerequisite for serious taxonomic endeavor. Others think that classifications of mental disorders may ignore subjective experience. A middle view holds that classification should at least map the contours of the phenomenology of mental illness. This paper examines these options. I conclude that it is not true that phenomenology is a logical prerequisite for classification, nor even that classification should necessarily respect phenomenological boundaries, but that detailed phenomenological examination can sometimes inform classification.
\end{abstract}

Keywords: Phenomenology, subjective experience, classification, D.S.M., I.C.D. 


\section{Introduction: Psychiatric classification and phenomenology.}

Two classifications of mental disorders dominate psychiatric research, practice and teaching; that included in the International Classification of Diseases (the I.C.D.), published by the World Health Organisation, and the Diagnostic and Statistical Manual of Mental Disorders (the D.S.M.), published by the American Psychiatric Association (W.H.O. 2007, A.P.A. 2000). The D.S.M. dominates in the United States, but in many parts of the world the I.C.D. is used by practitioners when diagnosing patients and for collecting health care statistics (Reed et al 2011). Worldwide, researchers are more likely to use D.S.M. categories to select subjects for research (López-Muñoz et al. 2008). In any event, the differences between the classifications are as nothing as compared to their similarities, and in this paper I will consider both classifications together.

A basic aim of the D.S.M. is to construct a classification that reflects the natural structure of the domain of mental disorder. When the D.S.M. is revised, a huge quantity of empirical research is reviewed with the aim of discovering where the boundaries between disorders should be drawn (as can be seen at http://www.dsm5.org/). The desire to produce an empirically-grounded classification in psychiatry is understandable. Classification can be powerful in science, so long as the categories included in a classification describe "natural kinds", that is divisions in nature that are grounded in the causal structure of reality. The Periodic Table in chemistry provides perhaps the best example of the potential power of classification. The Periodic Table provides a classificatory basis for chemistry that enables different types of stuff to be classified, and via this classification, for them to be understood and controlled. If mental disorders are natural kinds, we can hope that maybe one day psychiatric classification will ground psychiatric theory and practice in a way that approaches the successes of the Periodic Table in grounding chemistry (or perhaps, more plausibly, at least approaches the successes of classification in fields like botany or geology). At any event I take it that the ideal for classifications like the D.S.M. is to classify mental disorders in such a way that cases that are similar in their underlying causal mechanisms are classified together, while cases that are unalike are kept separate. The aims of the I.C.D. , an international classification originally developed to enable the collection of health statistics, are less straightforward. The mental disorders section of the I.C.D. is now closely tied to the D.S.M., however, as both the A.P.A. and the W.H.O. seek consistency between the classifications. It is thus fair to see both classifications as 
either directly or indirectly seeking to achieve an empirically-based classification of mental disorders that will provide a basis for psychiatric research and practice. ${ }^{1}$

Within psychiatry, the term "phenomenology" is used in a number of different ways. Sometimes it refers to any description of signs and symptoms. Sometimes it is used to mean any analysis of subjective experience. Sometimes its use is restricted to more specific practices associated with a particular school, for example, Husserlian or Jasperian phenomenology. My interests in this paper are fairly general, and I will consider the role that any type of analysis of subjective experience might play in informing classification.

Clearly reports of subjective experience must play at least some minimal role in psychiatry. Patients often request help because of troubling feelings, for example they may be sad or anxious. Here I aim to determine whether psychiatric classification needs to consider subjective experience in a way that goes beyond such minimal and common-sense reports. I want to know what role a detailed probing of subjective experience might play in the construction of classification schemes. At present the D.S.M. makes some limited use of phenomenology in this sense. For example, the criteria for mania include "flight of ideas or subjective experience that thoughts are racing” (A.P.A., 2000, p.362). The criteria for Borderline Personality Disorder include "feelings of emptiness" (A.P.A., 2000, p.710). Diagnoses of affective disorders may be modified by a melancholic features specifier, which refers to a "distinct quality of depressed mood (i.e. the depressed mood is experienced as distinctly different from the kind of feeling experienced after the death of a loved one)” (A.P.A., 2000, p.420). The descriptions of disorders included in the I.C.D. include similar references to subjective experience. These examples suggest that the D.S.M. and I.C.D. committees are at least open to the idea that a detailed analysis of subjective experience may be of relevance to psychiatric classification.

The aim of this paper is to consider what the relationship between psychiatric classification and the analysis of subjective experience should ideally be. This question is timely. The D.S.M. and I.C.D. are currently being revised (the new edition of the D.S.M. is due to be published in 2013, and the new I.C.D. in 2015). Some have claimed that producing a phenomenologically-informed descriptive psychopathology is a prerequisite for serious taxonomic endeavor. Is this true? Must a psychiatric classification scheme map the contours of the subjective experiences of mentally ill people? That is, must disorders that are experienced as alike be classified together, and those that are experienced as

\footnotetext{
${ }^{1}$ For criticisms of the idea that psychiatric classification might find natural kinds see Zachar 2000, Horwitz 2002. For a defence of the idea that psychiatric classification aims to find natural kinds see Cooper 2005, Murphy 2006.
} 
unalike be split-up? Could a successful classification of mental disorders completely ignore subjective experience?

In the remainder of the paper I will consider three claims of reducing strength

i. Phenomenology is a logical prerequisite for classification.

ii. Classification must respect phenomenological boundaries.

iii. Detailed phenomenological examination can sometimes inform classification

I will argue that i. and ii. are false, but that iii. is true.

\section{Is a detailed examination of subjective experience a necessary first step before classification can begin?}

One of the few papers that explicitly considers the links between phenomenology and psychiatric classification is Josef Parnas and Dan Zahavi (2002) "The role of phenomenology in psychiatric diagnosis and classification”. Parnas and Zahavi tell us that,

“...the majority of current diagnostic categories are based on typologies of human experience and behaviour...Therefore, a search for a faithful description of experience must be considered as a necessary first step in any taxonomic effort....” (Parnas \& Zahavi, 2002, p.137)

In the remainder of their paper, Parnass and Zahavi go on to outline their very particular views about the sort of phenomenology that might be required (they advocate phenomenology as practised by the Husserlian school). Here I will not consider the details of the proposal, but will focus on the basic claim that a detailed analysis of subjective experience is a necessary first step for classification. Parnas and Zahavi cite papers by Thomas Nagel and Karl Jaspers in support of this claim, so I will look first at arguments that might be found in these sources.

\section{Thomas Nagel}

Parnas and Zahavi take Thomas Nagel to have shown that "a necessary requirement for any coherent reductionism is that the entity to be reduced is properly understood" (Parnas and Zahavi, 2002, p.137; a similar claim is made by Sass and Parnas 2006, p.73). They think it follows that any 
psychiatric taxonomy, which has as its eventual aim the explanation of mental disorders in terms of brain processes, must be based on a detailed phenomenological analysis of mental disorder.

What exactly are Nagel's claims? In his paper “What is it like to be a bat?” (1974) Nagel argues against physicalism. The physicalist claims that all facts can be described in physical terms. However, Nagel argues, there will be some facts that will forever be beyond the reach of such analyses. These are facts about subjective experience. Plausibly, for example, there is something that it is like to be a bat. However, not being bats we cannot know what it is like to be a bat "from the inside". Nor, can we gain knowledge of this fact "from the outside", for example, via ever more detailed neurological studies on bats. Such knowledge necessarily misses out the "what it is likeness” of experience and thus misses out something essential.

Parnas and Zahavi cite Nagel to support the claim that in order to reduce phenomena one has to describe them fully. It is true that, in passing, Nagel does make remarks to this effect, but as I understand him this is not the key claim of Nagel's paper. On my interpretation, Nagel's argument rests specifically on the subjective nature of experience, and thus could not be generalised to make claims about reductionism in general. In any case, and whether Nagel believes it or not, the claim that one can only reduce a phenomenon once it has been fully described is not in general true. Consider what happens when the chemical structure of some substance is discovered. Reduction occurred when, for example, water was found to be $\mathrm{H}_{2} \mathrm{O}$, but chemists were still able to make new discoveries about the properties of water after this reduction.

Even if it isn't generally necessary to fully describe phenomena prior to them being reduced, could we entertain a weakened version of Nagel's thought that would apply specifically to psychopathology? Suppose we claim that the point of psychopathology is to understand the experiences of mentally ill people. In so far as a psychopathology without phenomenology would leave out the "what it is likeness" of mental illness, it would then miss the point of the endeavor. Such an argument may have some merit if one thinks that a central task for psychopathology is to supply us with an understanding of the experiences of mentally ill people. Of course, psychopathology might be defined in order to make this central to its aim by definition. However, competing definitions are possible. We might take psychopathology to be the science of classifying types of mental disorder in a way that will ground psychiatric research and practice, and take the main purpose of psychiatry to be the successful treatment of patients. On such a definition it is less clear why an in depth analysis of subjective experience will be essential. Maybe one day a classification on the basis of, say, neurological or genetic markers, will suffice for adequate 
treatment. Admittedly, one has to appreciate that patients are distressed to consider them candidates for treatment, but this requires no fine-grained phenomenological understanding.

At core, disagreement as to whether psychopathology must study the subjective experience of mental illness comes down to disagreement about the nature and aims of psychiatry. Nagel can only be used to argue that phenomenology is a necessary first step for classification if one already assumes that understanding subjective experience is essential for psychiatry, and this is a contestable assumption.

\section{$\underline{\text { Karl Jaspers }}$}

In the paper cited by Parnas and Zahavi, “The phenomenological approach in psychopathology” (1968 in translation, originally published 1912), Jaspers presents a vision for the development of psychopathology that has phenomenology at its core. He tells us that,

“...before real inquiry can begin it is necessary to identify the specific psychic phenomena which are to be its subject, and form a clear picture of the resemblances and differences between them and other phenomena with which they must not be confused. This preliminary work of representing, defining and classifying psychic phenomena, pursued as an independent activity constitutes phenomenology. The difficult and comprehensive nature of this preliminary work makes it inevitable that it should become for the time being an end in itself.” (Jaspers, 1968, p.1314).

Jaspers claims that phenomenological analysis of pathological experience is of key importance because psychiatric theorising had failed to reach any consensus. He hopes that phenomenology will enable an atheoretical collection of facts that can then be organised into a classification system and ground future research.

In the 1912 paper Jasper's does not present an explicit argument that phenomenology should ground classification in psychiatry, but rather presents a vision for the development of psychopathology that places phenomenology at its foundation. We only have reason to buy into this vision if the fruits of the approach can be demonstrated.

Given Jaspers' claims, one might expect his great work General Psychopathology to be clearly grounded in phenomenology. However, such an expectation will be disappointed. General 
Psychopathology went through multiple editions, with some changes being made between each one. In all editions, the text aims to summarise findings in psychopathology from a range of different viewpoints. Chapters set out what can be learnt from phenomenological study but also describe findings from very different sources, for example, research into the influence of the patient's environment, body shape and heredity. While the chapter on phenomenology comes first, Jasper's himself resists the claim that phenomenology is in any sense fundamental. He warns that

\footnotetext{
"It is wrong to call this book 'the principal text of phenomenology'. The phenomenological attitude is one point of view and one chapter has been devoted to it in some detail as the viewpoint is a new one. But the whole book is directed at showing that it is only one point of view among many and holds a subordinate position at that”. (Jaspers, 1997, p.48)
}

\begin{abstract}
I conclude that Jasper's work does not show that psychiatric classification must start with phenomenology.
\end{abstract}

\title{
Strengthening the argument
}

I have argued that the work of Nagel and Jaspers does not show that phenomenology is a necessary first step for psychiatric classification. Now I need to show that the claim that phenomenology is a necessary first step is not only unsupported by Nagel and Jaspers but is also untrue.

What does it mean to say that "a search for a faithful description of experience must be considered as a necessary first step in any taxonomic effort” (Parnas \& Zahavi, 2002, p.137)? It can mean only that it is inconceivable that any successful classification system could develop without taking a phenomenological analysis of mental disorder as a starting point. This is an extremely strong claim. To refute it all that is needed is a single example of a classificatory project that might have worked and that did not include phenomenological studies as a starting point. As our example, let's consider the project of classification by "pharmacological dissection” as most famously practiced by Donald Klein in the 1960s and 70s. Together with Max Fink and Max Pollack, Klein conducted studies which aimed to take patterns of response to different psychoactive drugs as the starting point for classification. Klein (1967) presents a study in which 311 patients, all of whom suffered from early and acute mental disorders, were randomly allocated to a course of either chlorpromazine, imipramine, or placebo. In the study "all referrals for psychotropic medication were accepted regardless of diagnosis or symptomatology, since it was hoped to be able to utilize the drug effects 
as tools for discriminating subgroups of patients” (Klein, 1967, p.118). The characteristics of drug responders were analysed. Various measures had predictive value, in particular, "the degree of retardation or excitation in the initial clinical state...[and]... the developmental history of the patient, with special emphasis upon signs of childhood deviancy” (Klein, 1967, p.119). Here we have a sketch of a classificatory project, that does not start with phenomenological analysis, and that might work; start with drug effects, analyse the characteristics of drug responders, classify patients into groups which might predict drug response.

Note here that I use Klein's study only to show that Parnas and Zahavi’s claim that "a search for a faithful description of experience must be considered as a necessary first step in any taxonomic effort” is untrue. I am not claiming that we should abandon phenomenological studies and embrace the method of pharmacological dissection instead. I am happy to concede that there may be many methods of constructing classification systems. Some start with phenomenology, but some do not.

\section{Must classification schemes respect phenomenological boundaries?}

Even if we need not start by considering subjective experience, might it be the case that a classification system should be revised to respect phenomenological boundaries when these are discovered? Must disorders that feel similar be classified together, and those that feel different be classified apart? I will argue that this suggestion, too, should be rejected.

I will argue for this via considering a specific case - masked depression. Masked depressions are supposed to be depressions that do not make people feel depressed. In outline, my argument will be as follows:

Premise 1: If the concept of masked depression makes sense then it is not necessary for psychiatric classification schemes to respect phenomenological boundaries.

Premise 2: The concept of masked depression does make sense.

Conclusion: It is not necessary for psychiatric classification schemes to respect phenomenological boundaries.

The idea that there might be masked depressions has a long history. Historically many of the claims made about depression can be traced back to older ideas about melancholia. Melancholia understood as a disorder of the black bile - was taken to be a condition that could manifest itself in a multitude of different ways (Radden 2003). Still, in so far as the causal root of all the conditions 
was the same, and it was thought that one form might morph into another, all were taken to be manifestations of the same condition.

Masked depression as a modern concept had its heyday in the pre-D.S.M.-III world of the 1970s. Papers on masked depression continue to be published - but mainly in French and German journals. Those who believe in masked depressions claim that cultural conditions can make it the case that certain individuals manifest depression in atypical ways. For example, in a society that sees sadness as unacceptable weakness, patients might complain instead of somatic complaints.

Why might one think that certain somatic symptoms are really depression? Psychiatrists writing on masked depression in the 1970s made use of the following sorts of evidence:

i. The incidence of typical depression varies around the world. Advocates of masked depression claim this is because depressed people in some cultures manifest depressive symptoms differently (Angst 1973).

ii. Family history studies suggest that while some relatives manifest typical depression, others manifest atypical depression (Pichot \& Hassan, 1973, p.71).

iii. Individual histories show shifts between typical and masked depression (Pichot \& Hassan, 1973, p.70).

iv. Somatic symptoms may vary periodically in ways characteristic of depressive symptoms, for example, they may be worse in the morning (Birkmayer, Neumayer and Riederer, 1973, p.158)

v. Somatic symptoms that are masked depression respond to antidepressants. (Pichot \& Hassan, 1973, p.72)

Here we will leave aside questions concerning the adequacy of such evidence and stick to considering whether the concept of masked depression makes sense. In "Is this Dame Melancholy? equating today's depression and past melancholia” Jennifer Radden (2003) points out that deciding what can count as “depression” depends on claims about the semantics of disease terms. On a causal account, a disease term refers to all conditions with a particular cause. On a descriptive account, a disease term refers to all conditions that fit a particular description. Only on a causal account, according to which “depression” refers to all disorders that have a certain underlying cause, can one sensibly claim that one disorder might manifest itself very differently in different cultures. On a descriptive account, according to which "depression” refers to disorders characterised by a characteristic set of symptoms, the idea that there might be forms of depression that manifest with atypical symptoms makes no sense. 
On Radden's account, a particular linguistic community (e.g. the community of mental health professionals) can choose whether to use a particular disease term in a descriptive or causal manner. Between 1980 (with the publication of the D.S.M.-III) and 1994 (with the publication of the D.S.M.-IV) psychiatric classification was officially descriptive. The D.S.M. committees felt that so little was known about the etiology of psychiatric disorders that theorising would be premature and that it was best to concentrate on a purely descriptive cataloging of conditions. Implicitly they thus chose to adopt a descriptive approach to disease terms. If one is being descriptive about diseases then one cannot talk of masked depression. And, indeed, the D.S.M.-III does not allow depression to be diagnosed in the absence of felt depression, claiming, "The essential feature is either a dysphoric mood, usually depression, or loss of interest or pleasure in all or almost all usual activities and pastimes” (A.P.A., 1980, p.210). However, since 1994, the claim to be atheoretical has been dropped from the D.S.M.. In the D.S.M.-IV someone can still only be diagnosed with depression if they feel depressed, or at least have a loss of pleasure. Still, with the descriptive approach abandoned there is now nothing in principle to prevent the inclusion of disorders like masked depression - i.e. disorders that are classified on the basis of their presumed causes, and where cases can be phenomenologically unalike but grouped together. The I.C.D. currently allows for the diagnosis of masked depression, under code F32.8 Other Depressive Episodes (W.H.O., 2007).

I accept that the theoretical claims on which the concept of masked depression is based may turn out to be false - maybe there are no masked depressions. The key claim here is that the idea that there may be masked depressions at least makes sense. We could adopt a causal approach to the semantics of disease-terms, and if we classify by causes, we can allow that a disorder might manifest in different ways - such that cases of the same disorder are phenomenologically quite unalike. I conclude that psychiatric classification need not respect phenomenological boundaries.

\section{Can detailed phenomenological analysis inform psychiatric classification?}

The claim that detailed phenomenological analysis can inform psychiatric classification is a weak claim. It says only that an analysis of subjective experience can sometimes be useful for psychiatric classification. Phenomenological differences will sometimes be informative about real causal distinctions. I suggest that this claim is plausibly true, as can be illustrated by considering a couple of examples. 
First, let's consider the relation between Borderline Personality Disorder (B.P.D.) and depression. People with B.P.D. suffer from "a pervasive pattern of instability of interpersonal relationships, selfimage, and affects, and marked impulsivity” (A.P.A. 2000, p.706). They also often feel depressed, and this raises a question about the relationship between depression and B.P.D.. Might B.P.D. be related to depression in some way?

Studies of phenomenology can contribute to answering this question. Several studies have suggested that the depressive feelings experienced in B.P.D. are phenomenologically distinct from those experienced in depression (Weston et al 1992, Levy et al 2007). In B.P.D., but not in depression, depressive feelings tend to be focussed on interpersonal worries, for example, they focus on fears of abandonment and rejection, a sense of emptiness and meaninglessness. This is evidence that B.P.D. and depression are distinct disorders. Other types of evidence are also relevant - family studies and patterns of medication response, for example, also suggest that B.P.D. and depression are distinct conditions (Gunderson 2009). My claim here is weak. Phenomenological evidence is evidence that can be considered alongside other evidence and can contribute to suggesting that the two conditions are distinct.

For our second example, let's consider research that has examined the prodromal features of schizophrenia. Sass and Parnas (2003) give an account of schizophrenia according to which it is at root a disorder of the sense of self. They see phenomenology as playing a special role in enabling apparently diverse symptoms to be unified and understood as forming a meaningful whole and they claim that their account can help to better define the prodromal features of schizophrenia. In particular, certain experiences that are indicative of problems with sense of self will be important for example, feelings of derealisation, not feeling oneself etc. This work provides another example of an area where a closer attention to phenomenology might prove fruitful. Sass and Parnas's work provides a hypothesis that might be studied, and if it does turn out that those who have early symptoms that are suggestive of problems with the sense of self go on to develop full-bloodied schizophrenia, such symptoms might be taken as early signs of the disorder.

\section{Conclusion}

This paper concerned a methodological question: to what extent is an analysis of subjective experience, including feelings and felt emotions, of importance for psychiatric classification? I considered three possible relationships: 
i. Phenomenology is a logical prerequisite for classification.

ii. Classification must respect phenomenological boundaries.

iii. Detailed phenomenological examination can sometimes inform classification.

I have argued that i. and ii. are false, but that iii. is true. Such conclusions are of importance because psychiatric classification forms a basis for psychiatric research and practice. Developing the best classification schemes requires various methodological issues to be solved. Figuring out the relationship between phenomenology and classification contributes to this project.

\section{Acknowledgements}

Versions of this paper were presented at the conference Emotion, Feeling and Psychopathology: Issues for Ongoing Research, held at Durham University, September 2010, and also at the Lancaster University philosophy work in progress seminar. I am grateful for the comments of those present, and also for comments from two anonymous referees for this journal.

\section{References}

American Psychiatric Association. (1980). Diagnostic and Statistical Manual of Mental Disorders (Third Edition) Washington: American Psychiatric Association.

American Psychiatric Association. (1994). Diagnostic and Statistical Manual of Mental Disorders (Fourth Edition) Washington: American Psychiatric Association.

American Psychiatric Association.(2000). Diagnostic and Statistical Manual of Mental Disorders (Fourth Edition Text Revision) Washington: American Psychiatric Association.

Angst, J. (1973). Masked depression viewed from the cross-cultural standpoint. In P. Kielholz (Ed.,) Masked Depression. (pp.269-274). Bern: Hans Huber Publishers. 
Birkmayer, W., Neumayer,E.\& Riederer, P. (1973). Masked depression in old age. In P. Kielholz (Ed.,) Masked Depression. (pp. 158-166). Bern: Hans Huber Publishers.

Cooper, R. (2005) Classifying Madness. Dordrecht: Springer.

Gunderson, J. (2009). Borderline Personality Disorder: Ontogeny of a Diagnosis. American Journal of Psychiatry, 166, 530-539.

Horwitz, A. (2002). Creating Mental Illness. University of Chicago Press: Chicago

Jaspers, K. (1912, 1968). The phenomenological approach in psychopathology. Translated and reprinted in British Journal of Psychiatry. 114, 1313-1323,

Jaspers, K. (1959, 1997). General Psychopathology. Translated from the German by J. Hoenig and M. Hamilton. Baltimore: John Hopkins Press.

Kielholz, P. (1973). Masked Depression. Bern: Hans Huber Publishers.

Klein, D. (1967). Importance of psychiatric diagnosis in prediction of clinical drug effects. Archives of General Psychiatry. 16, 118-126.

Klein, D. \& Fink, M. (1962). Psychiatric reaction patterns to imipramine. American Journal of Psychiatry, 119, 432-438.

Levy, K., Edell, W. \& McGlashan, T. (2007). Depressive experiences in inpatients with borderline personality disorder. Psychiatric Quarterly, 78, 129-143.

López-Muñoz, F., Garcia-Garcia, P., Sáiz-Ruis, J., Mezzich, J., Rubio, G., Vieta, E., Alamo, C. (2008). A bibliometric study of the use of the classification and diagnostic systems in psychiatry over the last 25 year. Psychopathology, 41, 214-225.

Murphy, D. (2006). Psychiatry in the Scientific Image. Cambridge, Massachusetts: MIT Press. 
Nagel, T. (1974). What is it like to be a bat? The Philosophical Review, 83, 435-450.

Parnas, J and Zahavi, D. (2002). The role of phenomenology in psychiatric diagnosis and classification. In M Maj, W. Gaebel, J. López-Ibor, N. Sartorius (Eds.) Psychiatric Diagnosis and Classification. (pp.137-162) Chichester: John Wiley and Sons.

Pichot, P and Hassan, J. (1973), Masked depression and depressive equivalents - problems of definition and diagnosis. In P. Kielholz (Ed.) Masked Depression. (pp. 61-76). Bern: Hans Huber Publishers.

Radden, J. (2003). Is This Dame Melancholy?: Equating today's depression and past melancholia. Philosophy, Psychiatry, \& Psychology. 10, 37-52,

Reed, G., Mendonça Corria, J., Esparza, P., Saxena, S., Maj, M. (2011). The WPA-WHO global survey of psychiatrists’ attitudes towards mental disorders classification. World Psychiatry, $10,118-131$.

Sass, L. and Parnas, J. (2003). Schizophrenia, Consciousness and the Self. Schizophrenia Bulletin, 29, 427-444.

Sass, L. and Parnas, J. (2006). Explaining schizophrenia: the relevance of phenomenology. In M. Chung, B. Fulford and G. Graham (Eds.) Reconceiving Schizophrenia. (pp.63-96). Oxford: Oxford University Press.

Weston, D., Moses, M., Silk, K., Lohr, N., Cohen, R. \& Segal, H. (1992). Quality of depressive experience in borderline personality disorder and major depression: when depression is not just depression. Journal of Personality Disorders, 6, 382-393.

W.H.O. (1993). The ICD-10 Classification of Mental and Behavioural Disorders Diagnostic Criteria for Research. Geneva: World Health Organisation. Available at http://www.who.int/classifications/icd/en/GRNBOOK.pdf

W.H.O. (2007) I.C.D.-10. Geneva: World Health Organisation. Available at http://www.who.int/classifications/icd/en/ 
Zachar, P. (2000). Psychological Concepts and Biological Psychiatry. Amsterdam: John Benjamin Publishing Co. 